\title{
Ionic and Thermal Transport in Na-Ion-Conducting Ceramic Electrolytes
}

\author{
Magnus Rohde ${ }^{1}$ (D) $\cdot$ ljaz U. I. Mohsin ${ }^{1} \cdot$ Carlos Ziebert $^{1} \cdot$ Hans Jürgen Seifert $^{1}$
}

Received: 5 May 2021 / Accepted: 24 June 2021 / Published online: 5 July 2021

(C) The Author(s) 2021

\begin{abstract}
We have studied the ionic and thermal transport properties along with the thermodynamic key properties of a Na-ion-conducting phosphate ceramic. The system $\mathrm{Na}_{1+\mathrm{x}} \mathrm{Al}_{x} \mathrm{Ti}_{2-x}\left(\mathrm{PO}_{4}\right)_{3}$ (NATP) with $x=0.3$ was taken as a NASICON-structured model system which is a candidate material for solid electrolytes in post-Li energy storage. The commercially available powder (NEI Coorp., USA) was consolidated using cold isostatic pressing before sintering. In order to compare NATP with the "classical" NASICON system, $\mathrm{Na}_{1+\mathrm{x}} \mathrm{Zr}_{2}\left(\mathrm{SiO}_{4}\right)_{x}\left(\mathrm{PO}_{4}\right)_{3-x}(\mathrm{NaZSiP})$ was synthesized with compositions of $x=1.7$ and $x=2$, respectively, and characterized with regard to their ionic and thermal transport behavior. While ionic conductivity of the NaZSiP compositions was about more than two orders of magnitude higher than in NATP, the thermal conductivity of the NASICON compound showed an opposite behavior. The room temperature value was about a factor two higher in NATP compared to NaZSiP. While the thermal conductivity decreases with increasing temperature in NATP, it increases with increasing temperature in NaZSiP. However, the overall change of this thermal transport parameter over the measured temperature range from room temperature up to $800{ }^{\circ} \mathrm{C}$ appeared to be relatively small.
\end{abstract}

Keywords Ceramic $\cdot$ Solid electrolyte $\cdot$ Thermal conductivity $\cdot$ Thermal diffusivity

\section{Introduction}

Solid ionic conductors are crucial components [1-3] of all solid-state batteries since they are transporting ions in electrochemical cells from the cathode to the anode and vice versa. The main advantage compared to conventional cells [4] is the lack of a liquid organic electrolyte, which is often considered as a safety risk [5] due to their high chemical reactivity causing thermal run-away events at higher temperatures.

Magnus Rohde

magnus.rohde@kit.edu

1 Institute for Applied Materials - Applied Materials Physics (IAM-AWP), Karlsruhe Institute of Technology (KIT), 76344 Eggenstein-Leopoldshafen, Germany 
Since the last decade Li-ion cells containing liquid electrolytes are dominating the market of portable and stationary devices for electrochemical storage. However, up to now all solid-state cells are only available as prototypes on the laboratory scale. Furthermore, due to the expected increasing number of Li-ion cells for the growing market of electrical vehicles and stationary storage for intermittent renewable energy sources, limited Lithium resources and higher costs may call for alternative elements in future decades. A promising alternative at least for stationary storage could be Sodium [6,7], which is cost effective without the limitation in the Li resources and exhibits comparable physicochemical and electrochemical properties.

Phosphate-based ceramic electrolytes are promising candidates [8-10] for allsolid-state Na-ion cells. Specifically, the NASICON (Na Super Ionic Conductor) structured electrolytes can be tailored to required properties by doping and structural tuning. The NASICON framework is composed of metal oxide $\mathrm{MO}_{6}$-octahedra which are connected to $\mathrm{SiO}_{4}$ - and $\mathrm{PO}_{4}$-tetrahedra, respectively. Within this $3 \mathrm{D}$ structure, the $\mathrm{Na}^{+}$ions are migrating by hopping from occupied to empty lattice sites along the c-axis. In NATP (space group R $\overline{3} \mathrm{c}$ ), the Na ions are located at two different sites with Na1 (Wyckoff position 18e), which is nearly empty, and Na2 (Wyckoff position $6 \mathrm{~b}$ ), which is fully occupied. In NaZSiP (space group $\mathrm{C} 2 / \mathrm{c}$ ), the $\mathrm{Na}$ ions are distributed over three different sites: Na1 (Wyckoff position 4d) has an occupation level of $80 \%, \mathrm{Na} 2$ (Wyckoff position 4e) is completely occupied, and Na3 (Wyckoff position 8f) is occupied to $60 \%$.

Although a large number of studies is published on the relationship between the ionic transport and the structural and morphological properties [11-18] in phosphate-based Na-ion conductors, up to now the number of publications [19-22] dealing with the thermophysical or thermodynamic properties is significantly lower. Up to now, to the authors best knowledge, investigations which studied the ionic conductivity along with the thermal conductivity are still missing. Therefore, a possible correlation between the ionic and the thermal transport remains an open question due to the lack of combined ionic and thermal transport data, respectively.

Therefore, our research work within this study is focused on the determination of the thermophysical properties like the thermal conductivity and the specific heat capacity and also on the evaluation of possible interrelations between mechanisms of ionic and thermal transport as well as thermodynamic properties of ceramic $\mathrm{Na}^{+}$ ion conductors.

\section{Experimental}

Commercially available NATP powder (NEI Coorp., USA) with a stoichiometry of $\mathrm{Na}_{1.3} \mathrm{Al}_{0.3} \mathrm{Ti}_{1.7}\left(\mathrm{PO}_{4}\right)_{3}$ was compacted to a green state using Cold Isostatic Pressing (CIP) at pressures of about $300 \mathrm{MPa}$. After a detailed in situ sintering study in order to optimize the heat treatment during the consolidation, NATP samples were sintered at $900{ }^{\circ} \mathrm{C}$ with a holding time of $11 \mathrm{~h}$ which results in a density of $2.86 \mathrm{~g} \cdot \mathrm{cm}^{-3}$, which is $96 \%$ of the theoretical density. X-ray powder diffraction (XRD) confirmed the rhombohedral lattice structure $(\mathrm{R} \overline{3} \mathrm{c})$ of NATP. By chemical analysis it could be 
shown that the NATP is nearly phase pure with only a small amount of $\mathrm{AlPO}_{4}$ as impurity phase, which was less than $2.5 \mathrm{wt} \%$, located in the grain boundaries.

Since there is no commercially available source for $\mathrm{Na}_{1+\mathrm{x}} \mathrm{Zr}_{2}\left(\mathrm{SiO}_{4}\right)_{\mathrm{x}}\left(\mathrm{PO}_{4}\right)_{3-\mathrm{x}}$ (NaZSiP), a synthetization route had to established for the production of the starting powder. Since a simple solid-state reaction process is known to be problematic $[13,23,24]$ achieving the target stoichiometry due to the high-temperature processes involved, the so-called solution-assisted solid-state reaction (SA-SSR) [25] was adapted for the production of the NaZSiP powder. For the consolidation of the ceramic powder, a field-assisted sintering technology (FAST) at a temperature of $900{ }^{\circ} \mathrm{C}$ and also CIP followed by pressureless sintering at $1250{ }^{\circ} \mathrm{C}$ for $5 \mathrm{~h}$ was applied. Structural analysis by XRD confirmed the NASICON structure which shows a monoclinic distortion, and also traces of ${\mathrm{Z} \mathrm{ZO}_{2}}_{2}$ phase could be found.

The ionic conductivity was determined on the sintered samples by impedance spectroscopy in the frequency range from $100 \mathrm{~Hz}$ to $10 \mathrm{MHz}$ as a function of temperature from RT up to $200{ }^{\circ} \mathrm{C}$. The cylindrical sample with a typical thickness of $1 \mathrm{~mm}$ and a diameter of $10-12 \mathrm{~mm}$ is mounted in a heated ceramic sample holder which is built in a shielded chamber. Before the measurements, Au contacts with a thickness of about $150 \mathrm{~nm}$ are sputtered onto both faces of the sample. The voltage and current are applied by Au-coated spring-loaded contact pins in a two-point arrangement. The temperature is controlled by a K-type thermocouple located close to the sample. The temperature stability of the system is better than $1{ }^{\circ} \mathrm{C}$.

The impedance is measured using an excitation AC voltage with a small amplitude $\left(\mathrm{V}_{\mathrm{A}}=100 \mathrm{mV}\right)$ at a given frequency $f$. The voltage $V$ can be expressed as follows[26]:

$$
V(t)=V_{A} \cdot \sin (2 \pi f t)
$$

In a linear system, the response current signal $I(t)$ has an amplitude $I_{\mathrm{A}}$ and is shifted in phase:

$$
I(t)=I_{A} \cdot \sin (2 \pi f t+\varphi)
$$

The complex impedance can be calculated as the ratio of the input voltage $\mathrm{V}(\mathrm{t})$ and the measured current $I(t)$ :

$$
Z=\frac{V(t)}{I(t)}=\frac{V_{A} \sin (2 \pi f t)}{I_{A} \sin (2 \pi f t+\varphi)}=Z_{A} \frac{\sin (2 \pi f t)}{\sin (2 \pi f t+\varphi)}
$$

The impedance can be, therefore, expressed in terms of a magnitude or absolute value $Z_{\mathrm{A}}=|Z|$ and a phase shift $\varphi$. Alternatively, it can be written as a sum of a real $Z^{\prime}$ and an imaginary part $Z^{\prime \prime}$, respectively:

$$
Z=Z^{\prime}+i Z^{\prime \prime}
$$

The impedance spectrum is recorded by a precision LCR meter (LCR 8110, GW Instek) over the frequency range given above. The results are plotted in the complex plane (cole-cole plot) which can be used for a first estimate of the bulk resistance by an inspection of the diameter of the depressed semicircle. For a more accurate 
evaluation, the experimental data are fitted to an equivalent circuit model (ECM). The real part of the frequency-dependent conductivity $\sigma^{\prime}$, which is an important parameter for the ion migration in solid electrolytes [27], is calculated using the following expression:

$$
\sigma^{\prime}=\frac{Z^{\prime}}{Z^{\prime 2}+Z^{\prime \prime 2}} \cdot \frac{d}{A}
$$

with $\mathrm{d}$ and A the thickness and surface area of the sample, respectively.

For each material, three different samples are taken from the same synthesis. The recording of the impedance spectra was repeated three times for each temperature level. The standard uncertainties of the bulk resistance and ionic conductivity are evaluated according to the guidelines of GUM (Guide to the Expression of Uncertainty in Measurements) [28].

In addition to the ionic transport also the thermal transport properties were measured by a Laser Flash Apparatus (LFA 427, Netzsch GmbH) on the sintered samples, which enables the direct determination of the thermal diffusivity. This particular property can be converted to the thermal conductivity if the specific heat and the density of a sample are known. Furthermore, changes of the thermal diffusivity within a given material system can be directly related to a variation in the phonon mean free path [29], which is affected by scattering processes at grain and phase boundaries, respectively, but also by defects. This is important for solid electrolytes since the number and distribution of point defects i.e., vacancies are crucial for the transport of ions within a crystal structure.

In a Laser Flash measurement [30], a short laser pulse $\left(\mathrm{t}_{\mathrm{pulse}}=1 \mathrm{~ms}\right)$ is directed onto the front face of a cylindrical sample and the temperature rise is recorded as a function of time on the rear face by an infrared detector. Under ideal conditions, the thermal diffusivity $\alpha$ can be calculated from the time-temperature history by the following:

$$
\alpha=0.1388 \cdot \frac{d^{2}}{t_{0.5}}
$$

where $\mathrm{d}$ is the thickness of the sample and $\mathrm{t}_{0.5}$ the so-called half max time, i.e., the time in which the temperature rise on the rear face reaches half of the maximum value. Since this simple model expression is only valid under adiabatic conditions, a large number of more sophisticated models [31] have been developed which are able to consider deviations from ideal conditions by fitting the complete temperature-time curve. In this work a data reduction algorithm is used which is able to include radiation heat losses and finite size effects within the so-called combined model [32].

For the measurement the sample is placed in heating chamber which can be operated under vacuum or inert gas in a temperature range from RT to $1500{ }^{\circ} \mathrm{C}$. Before the measurement the faces of the sample are coated with thin graphite film with a typical thickness of $10 \mu \mathrm{m}-20 \mu \mathrm{m}$. This is necessary since the ceramic samples are translucent for the infrared radiation of the pulsed laser (Nd:YAG, $1064 \mathrm{~nm}$ ) and for the $\mathrm{InSb}$ detector which records the temperature rise on the rear face of the sample. 
The temperature stability of the furnace system is better than $1.5^{\circ} \mathrm{C}$. At each given temperature level the flash measurements were repeated for six times. Three different sample pieces from the same synthesis were included in a complete measurement run. The data were analyzed according to the GUM guidelines [28] in order to evaluate the standard uncertainty.

The specific heat capacity of the densified, sintered NASCION materials was measured using the standard three-step procedure [33] in a differential scanning calorimeter DSC 404 (Netzsch GmbH, Germany) for the high-temperature range from $220{ }^{\circ} \mathrm{C}$ to $900{ }^{\circ} \mathrm{C}$ and DSC 204 (Netzsch GmbH, Germany) for the low-temperature range from $25^{\circ} \mathrm{C}$ to $320^{\circ} \mathrm{C}$.

The principle of differential scanning calorimetry (DSC) is based on the highprecision measurement of the heat flow during a predefined temperature cycle. During a "temperature scan", the temperature difference between a sample and a reference crucible placed symmetrically on a disk-type sample holder surrounded by a furnace is recorded. The heat flow rate between the crucibles can be determined as a function of temperature and time. For the measurement of the specific heat capacity [34], three "temperature scans" with identical heating rates, start, and end temperatures, respectively, are necessary. In the first step, a baseline is recorded with two empty crucibles. In the second and third run, the heat flow rates are recorded with the sample and a standard reference material placed in the sample crucible. Using data of the heat flow rate of the three scans, the specific heat $c_{p}$ of the sample can be calculated as follows:

$$
C_{p}=\frac{\Phi_{S}-\Phi_{B}}{\Phi_{R}-\Phi_{B}} \cdot \frac{m_{R}}{m_{S}} \cdot C_{R}
$$

where $\Phi_{B}, \Phi_{R}$, and $\Phi_{S}$ are the heat flow rates of the baseline, reference, and sample run, respectively, $m_{R}$ and $m_{S}$ mass of the reference and the sample, and $C_{R}$ is the specific heat capacity of the reference material. Synthetic sapphire (NIST, SRM 720 [35]) is used as a reference material.

In this work three different samples from the same batch were used for the heat capacity measurements. A typical sample size is $5 \mathrm{~mm}$ in diameter, with a thickness less than $1 \mathrm{~mm}$ and a typical sample mass of about $40 \mathrm{mg}$. The bottom side of the sample is polished in order to achieve a good contact to the crucible. For every sample, the temperature scan was repeated for three times resulting in three heating and three cooling ramps. Between each heating or cooling ramp, an isothermal phase with a duration of $30 \mathrm{~min}$ was introduced in order to establish thermal equilibrium before the next ramp. During the measurement, the furnace chamber and the sample holder are purged with high-purity Argon gas. Al crucibles are used in the lowtemperature DSC204 and Pt crucibles for the high-temperature part in the DSC404. The data from the three samples and the three heating ramps are used to evaluate the standard uncertainty of the specific heat according to GUM [28].

The density of the sintered samples was measured by the Archimedes method. This is also called hydrostatic weighing since the weight of the sample is measured in air and also immersed in a liquid. From the both measurements, the density $\rho$ of a sample can be calculated as follows: 


$$
\rho=\rho_{\text {liq }} \cdot \frac{m_{\text {air }}}{m_{\text {air }}-m_{\text {liq }}}
$$

where $\rho_{\text {liq }}$ is the density of the liquid, $\mathrm{m}_{\text {air }}$ and $\mathrm{m}_{\text {liq }}$ are the masses measured in air and in the liquid, respectively. In this work, high-purity ethanol was used a liquid with a density of $0.789 \mathrm{~g} \cdot \mathrm{cm}^{-3}$. For the determination of the masses, a high-precision balance (Mettler Toledo MS-XSR105) is used which is equipped with an additional density measurement kit (MS-DNY-54) which allows the weighing in air and in the liquid without any additional sample preparation or changes in the balance setup. A typical sample mass varies between $600 \mathrm{mg}$ and $1.3 \mathrm{~g}$. Three different samples from the same batch are used in the density measurement. Each measurement was repeated for three times. The standard uncertainty of the density values was evaluated according to the guidelines of GUM [28].

\section{Results and Discussion}

Typical results extracted from the impedance spectroscopy on NATP are shown in Fig. 1. In the complex plane $Z^{\prime \prime}$ vs. $Z^{\prime}$ a single depressed semicircle is visible. The bulk resistance can be estimated by measuring the diameter of the semicircle. This estimate is then further on used as an initial value for the fit procedure based on an equivalent circuit model (ECM). The ECM used for the fitting of the experimental data is shown in the inset of Fig. 1 (right). It consists of a combination of a resistance $\mathrm{R}_{\mathrm{SE}}$ representing the bulk resistance of the electrolyte in parallel to a constant phase element $\mathrm{CPE}_{\mathrm{SE}}$ for the bulk capacitance and an additional $\mathrm{CPE}_{\mathrm{BLE}}$ for the blocking electrodes [26, 27].

The ECM fit shown as a continuous line in Fig. 1 yields a value for the bulk resistance $\mathrm{R}_{\mathrm{SE}}=1.5 \pm 0.05 \times 10^{-6} \Omega$ and for the conductivity $\sigma^{\prime}=2.7 \pm 0.095 \times 10^{-7}$ $\mathrm{S} \cdot \mathrm{cm}^{-1}$. The uncertainties were calculated according to the guidelines of GUM [28] with a coverage factor of $k=2$.
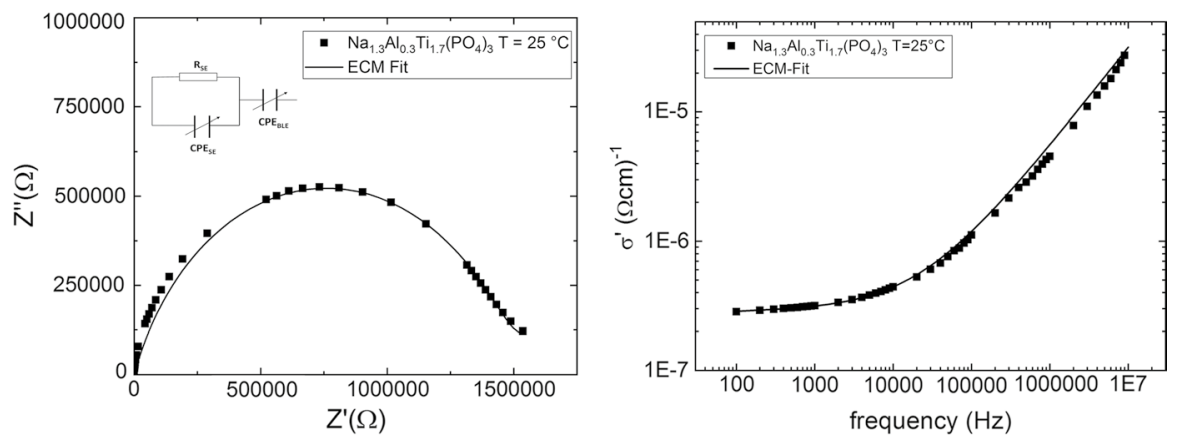

Fig. 1 Left: Cole-Cole presentation of the complex impedance, right: real part of the conductivity as a function of the frequency 
The experimental data of the measured ionic conductivity in Fig. 2 are plotted in a $\log$ scale vs $1000 / \mathrm{T}$ in order to determine the activation energy $\mathrm{E}_{\mathrm{a}}$ for the ionic transport. The lines are the results of a calculation of the ionic conductivity $\sigma$ vs. $\mathrm{T}^{-1}$ using the Arrhenius equation:

$$
\sigma=\frac{\sigma_{0}}{T} \cdot e^{-\frac{E a}{k_{B}} \cdot T}
$$

where $\sigma_{0}$ is the pre-exponential factor and $\mathrm{E}_{\mathrm{a}}$ the activation energy, which have been used as fitting parameters in Eq. 9 to fit the experimental data. The uncertainties of the measured ionic conductivity were estimated to be about $\pm 3.5 \%$. For the activation energy, a standard deviation of $\pm 5 \%$ was derived from the fitting procedure.

The two NASICON systems differ strongly in the ionic conductivity although the values of the activation energy $\mathrm{E}_{\mathrm{a}}$ are comparable. The room temperature value of NATP is $\sigma_{\text {Ion }}=2.7 \times 10^{-7}(\Omega \cdot \mathrm{cm})^{-1}$ being significantly below the level of $10^{-4}$ $(\Omega \cdot \mathrm{cm})^{-1}$ in NaZSiP. The ionic conductivity increases by more than three orders of magnitude from NATP to NaZSiP. However, the activation barrier for ion transport, i.e., the activation energy, is only lowered by a factor of two.

This large difference in the ionic conductivity comparing the NATP $\left(\mathrm{Na}_{1.3} \mathrm{Al}_{0.3} \mathrm{Ti}_{1.7}\left(\mathrm{PO}_{4}\right)_{3}\right)$ with $\mathrm{Na}_{2.7} \mathrm{ZSiP}$ and further to $\mathrm{Na}_{3.0} \mathrm{ZSiP}$ (i.e., $\left.\left.\mathrm{Na}_{1+x} \mathrm{Zr}_{2}\left(\mathrm{SiO}_{4}\right)_{x}\left(\mathrm{PO}_{4}\right)_{3-x}\right), x=1.7-2.0\right)$ is not surprising at a first glance since the $\mathrm{Na}$ content rises from 1.3 over 2.7 to 3.0 per formula unit, which increases also the number of $\mathrm{Na}+$ charge carriers within the structure. Together with the increased $\mathrm{Na}$ concentration additional sites appear for the $\mathrm{Na}$ ions which are only partially occupied. This effect is important for the ion migration through the structure since a balanced configuration of occupied and vacant lattice sites is crucial for ionic conduction. In NASICON systems the distance between two Na sites is about $3 \AA$ depending on the

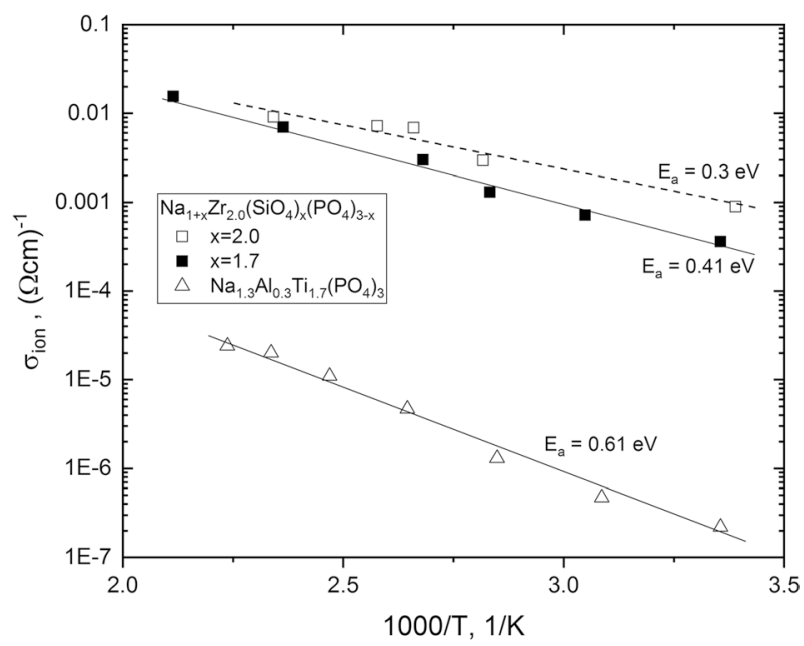

Fig. 2 Ionic conductivity of the NASICON systems as a function of the inverse temperature 
specific composition. The ion transport occurs by thermal activation which explains the increasing conductivity within a given system with increasing temperature.

Our conductivity data can be compared with the literature values of NASICON systems. Mouahid et. al. [36] synthesized a series of NATP ranging from $0<x<0.9$ using a solid-state reaction. For the lowest $\mathrm{Na}$ concentration of $\mathrm{x}=0.4$, they determined a conductivity of $\sigma_{25}{ }^{\circ} \mathrm{C}=5.6 \times 10^{-8} \mathrm{~S} \cdot \mathrm{cm}^{-1}$ and an activation energy of $\mathrm{E}_{\mathrm{a}}=0.54 \mathrm{eV}$. At a composition with $\mathrm{x}=0.9$, they found a room temperature conductivity of $1.3 \times 10^{-7} \mathrm{~S} \cdot \mathrm{cm}^{-1}$ and an activation energy of $0.51 \mathrm{eV}$. While the ionic conductivity was found to be lower than our values the activation energy is significantly lower. However, this can be explained by a sintering process at lower temperatures compared to our heat treatment. Lower sintering temperatures may lead to higher porosities which reduces the ionic conductivity.

Nieto-Munez and co-workers [12] synthesized glass-ceramics of NATP $\left(\mathrm{Na}_{1+\mathrm{x}} \mathrm{Al}_{\mathrm{x}} \mathrm{Ti}_{2-\mathrm{x}}\left(\mathrm{PO}_{4}\right)_{3}\right.$ with $\left.0<x<1.4\right)$ by a melt-quenching route. The glass transition as well as crystallization temperature were determined in a DSC and later heat treated the material at the onset of the crystallization temperature which was determined to be around $700{ }^{\circ} \mathrm{C}$. The room temperature values of the ionic conductivity are comparable with the corresponding conductivity data of this work while the activation energy is slightly lower with a value of $0.54 \mathrm{eV}$ for the composition of $\mathrm{Na}_{1.4} \mathrm{Al}_{0.4} \mathrm{Ti}_{1.6}\left(\mathrm{PO}_{4}\right)_{3},(x=0.4)$ which is closest to our studied composition with $x=0.3$.

In the "classical" NASICON system $\mathrm{Na}_{3} \mathrm{Zr}_{2}\left(\mathrm{SiO}_{4}\right)_{2}\left(\mathrm{PO}_{4}\right)$, the landscape of possible ionic conductivity values seems to be much wider [11, 13, 23]. Depending on the exact composition, processing route, and the heat treatment, room temperature values ranging from $4 \times 10^{-4} \mathrm{~S} \cdot \mathrm{cm}^{-1}$ up to about $1 \mathrm{mS} / \mathrm{cm}$ are found, while the activation energy is typically around $0.3 \mathrm{eV}$. The conductivity data of this work are within this range.

The measured thermal diffusivity values of NaZSiP and NATP are shown in Fig. 3. Standard uncertainties were calculated with a coverage factor of $k=2$. It is obvious from the diagram that the uncertainties are higher in NATP than in the NaZSiP materials. This effect can be often observed with increasing thermal diffusivity values which leads to a lower signal to noise ratio in the laser Flash signal [31] inducing a higher scatter of the measured data. However, the relative uncertainty is about $\pm 3 \%$ for NaZSiP and NATP which is typical in a Laser Flash apparatus. The thermal diffusivity values decrease with increasing temperature following a relationship of $\alpha \propto T^{-p}$ with $p=0.17$ for NATP and $p=0.04$ for both NaZSiP compositions. Typical values for technical ceramics are in the range $0.5<p<1.0[29,37]$ depending on the density of defects. This very low value of the exponent $\mathrm{p}$ reflects the strong influence of the defect scattering on the thermal diffusivity. Although the variation of the thermal diffusivity is relatively small within the temperature range of RT up to $900{ }^{\circ} \mathrm{C}$, its thermal behavior is opposite to the ionic conductivity which shows a strong increase between RT and $200{ }^{\circ} \mathrm{C}$ by about two orders of magnitude. Furthermore, the NATP system with the lowest ionic conductivity exhibits the highest thermal diffusivity values compared to NaZSiP over the whole temperature range. Since the thermal diffusivity is directly related to the phonon mean free path, this gives some insights into the structure property relationship of ionic conductors, 


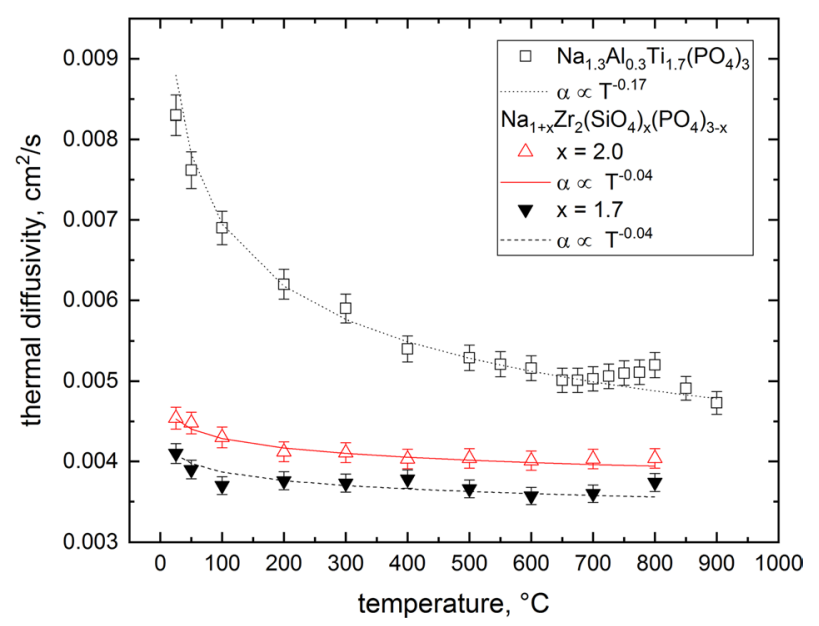

Fig. 3 Thermal diffusivity $\alpha$ of the NASICON in comparison to NATP systems as a function of temperature

specifically in Na-conducting NASICON systems. Obviously, the Na concentration and the vacancy distribution are exposing an opposite effect on the ionic and thermal transport, respectively. While both are crucial for a high ionic conductivity and a low activation energy, they are perturbing the phonon transport with an effect not only on the level or absolute value of the thermal diffusivity but also on its temperature variation.

The heat capacity data of the studied NASICON systems are shown in Fig. 4. The relative uncertainties (coverage factor $k=2$ ) are about $\pm 3.5 \%$ in the low-temperature

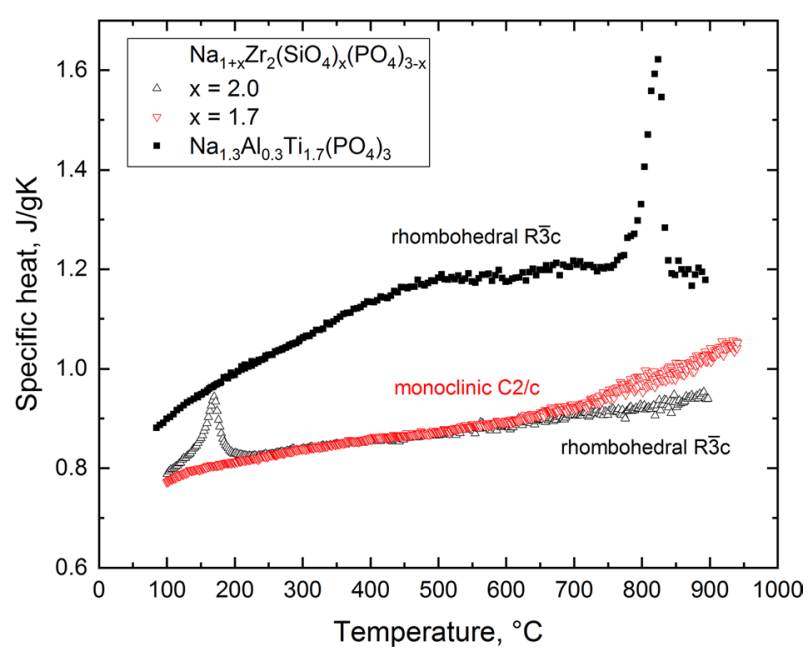

Fig. 4 Specific heat capacity Cp of the NASICON and NATP systems 
region, but increases up to $\pm 4.5 \%$ at temperatures above $700{ }^{\circ} \mathrm{C}$. In NATP, the apparent heat capacity is continuously growing with increasing temperature up to about $750{ }^{\circ} \mathrm{C}$. Above this temperature, a peak centered around $800{ }^{\circ} \mathrm{C}$ is visible, which can be related to phase transformations in NATP. The specific nature of these transformations has to be evaluated in further studies, since no comparable data can be found in the literature. However, it should be noted here that a similar behavior was also observed in other phosphate-based $\mathrm{Li}+$ and $\mathrm{Na}+$-conducting systems $[38,39]$, which could be at least partially explained by a rotational distortion of the $\mathrm{MO}_{6}$-octahedra. This effect causes a rotation of the $\mathrm{PO}_{4}$-tetrahedra within the flexible polyhedral framework. The degree of distortion and the induced rotation depends obviously on the $\mathrm{Na}^{+}$doping level and as well as on the occupation of the different $\mathrm{Na}$ sites, respectively.

The two NaZSiP compositions show differences in the specific heat data below $300{ }^{\circ} \mathrm{C}$ and above $700{ }^{\circ} \mathrm{C}$, while in between both systems can be characterized by nearly the same specific heat values. The enthalpy excursion in the apparent specific heat in the temperature range $100{ }^{\circ} \mathrm{C}<\mathrm{T}<200{ }^{\circ} \mathrm{C}$ of $\mathrm{Na}_{3.0} \mathrm{ZSiP}$ is due to structural phase transformation $[19,20,40]$ from the monoclinic $\mathrm{C} 2 / \mathrm{c}$ to the rhombohedral $\mathrm{R} \overline{3} \mathrm{c}$ structure. This transformation cannot be observed in the $\mathrm{Na}_{2.7} \mathrm{ZSiP}$ composition since it stays within the monoclinic $\mathrm{C} 2 / \mathrm{c}$ structure over the whole temperature range. However, at temperature above $750{ }^{\circ} \mathrm{C}$, the specific heat capacity values as a function of temperature of $\mathrm{Na}_{2.7} \mathrm{ZSiP}$ are increasing with a higher slope compared to $\mathrm{Na}_{3.0} \mathrm{ZSiP}$. This additional anharmonic contribution could be due to an increased thermal expansion or further vacancy formation.

Studies of the specific heat capacity in Li-conducting NASICON systems, namely, LAGP $\mathrm{Li}_{1+\mathrm{x}} \mathrm{Al}_{\mathrm{x}} \mathrm{Ge}_{2-\mathrm{x}}\left(\mathrm{PO}_{4}\right)_{3}$ [41] with different compositions showed similar high-temperature behavior. However, the LAGP exhibited the anharmonic contributions for all studied compositions.

The values of the thermal conductivity $\lambda$ of NATP and the NaZSiP compositions were calculated using the data of the thermal diffusivity $\alpha$, the heat capacity $\mathrm{Cp}$, and the density $\sigma$, respectively, by the following equation:

$$
\lambda=\alpha \cdot C_{p} \cdot \rho
$$

For the calculation with Eq. 10, the specific heat data $\mathrm{Cp}(\mathrm{T})$ were taken from the experimentally determined data ignoring the peaks due to the phase transitions, where extrapolated baseline values were used. The measured density values at room temperature are given in Table 1. The density was not corrected due to thermal

Table 1 Data of the density and elastic modulus used for the evaluation of the sound velocity and the phonon mean free path

\begin{tabular}{lllll}
\hline & $\begin{array}{l}\text { Density } \\
\left(\mathrm{g} \cdot \mathrm{cm}^{-3}\right)\end{array}$ & $\begin{array}{l}\text { Elastic modulus } \\
(\mathrm{GPa})\end{array}$ & $\begin{array}{l}\text { Sound velocity } \\
\left(\mathrm{m} \cdot \mathrm{s}^{-1}\right)\end{array}$ & $\begin{array}{l}\mathrm{l}_{\mathrm{Ph}} \\
(\tilde{\mathrm{A}})\end{array}$ \\
\hline $\mathrm{Na}_{1.3} \mathrm{Al}_{0.3} \mathrm{Ti}_{1.7}\left(\mathrm{PO}_{4}\right)_{3}$ & $2.64 \pm 0.02$ & $90 \pm 4.6$ & $5.84 \times 10^{3}$ & 4.6 \\
$\mathrm{Na}_{2.7} \mathrm{Zr}_{2}\left(\mathrm{SiO}_{4}\right)_{1.7}\left(\mathrm{PO}_{4}\right)_{1.3}$ & $3.07 \pm 0.03$ & $73.4 \pm 4.2$ & $4.89 \times 10^{3}$ & 2.6 \\
$\mathrm{Na}_{3.0} \mathrm{Zr}_{2}\left(\mathrm{SiO}_{4}\right)_{2}\left(\mathrm{PO}_{4}\right)$ & $3.17 \pm 0.02$ & $64.5 \pm 3.9$ & $4.51 \times 10^{3}$ & 3.0 \\
\hline
\end{tabular}


expansion, since the volume expansion in NATP and NaZSiP [42, 43] is less than $1 \%$ at $900{ }^{\circ} \mathrm{C}$. The results of the thermal conductivity as a function of the temperature from RT to $900{ }^{\circ} \mathrm{C}$ are shown in Fig. 5. The combined standard uncertainty of the thermal conductivity was calculated considering the uncertainties of the thermal diffusivity, the heat capacity, and the density, respectively.

The temperature variation of $\lambda(T)$ in NATP is very small. It varies only by $15 \%$ over the whole temperature range. However, $\lambda(T)$ decreases with increasing temperature, but temperature variation of thermal conductivity is even lower than that of the thermal diffusivity, which is due to the effect that the specific heat increases toward higher temperatures and compensates the stronger decrease of the thermal diffusivity. The physical effect behind this behavior is the strong phonon scattering by point defects in the NATP, which leads to a low and nearly temperature-independent phonon mean free path.

In the NaZSiP compositions, a different temperature-dependent behavior can be observed. Although, the variation of $\lambda(T)$ over the investigated temperature range is also small, the values tend to increase with increasing temperature. While the variation of the thermal diffusivity with temperature is much smaller in NaZSiP than in NATP, the monotonic growth of the specific heat data with temperatures determines the behavior of the thermal conductivity and leads to increasing thermal conductivity values with increasing temperatures.

It should be noted here that a $1 / \mathrm{T}$ behavior of the thermal conductivity $[36,37]$, i.e., an exponent close to -1, is typically expected in non-conducting ceramic materials. Therefore, it can be concluded that a small temperature exponent tending to zero can obviously be taken as a kind of signature for superionic conductors, which results from a high concentration of point defects, which is needed for the thermally activated transport of the ions. For the further discussion, it might be helpful to take a closer look on the relationship of the thermal conductivity to the phonon mean

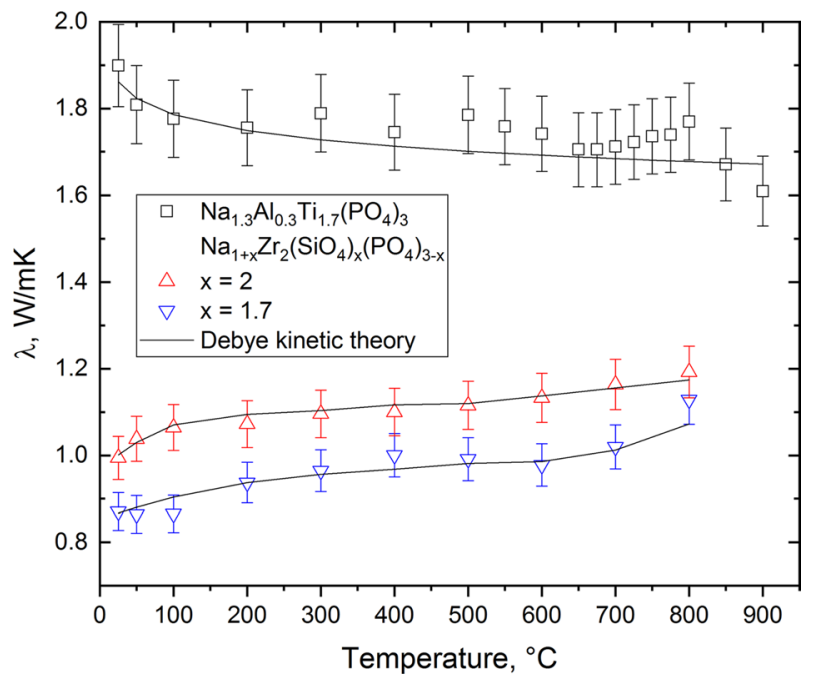

Fig. 5 Thermal conductivity $\lambda$ of NATP and NaZSiP as a function of the temperature 
free path which is given by the following equation derived from the Debye kinetic theory of gases [44]:

$$
\lambda=\frac{1}{3} \cdot C \cdot v \cdot l_{P h}
$$

where $\mathrm{C}$ is the heat capacity per unit volume, $\mathrm{v}$ is the average phonon group velocity, and $l_{P h}$ is the mean free path of the phonons between scattering events. It is obvious that the thermal diffusivity $\alpha$ can be also related to the phonon mean free path as follows:

$$
\alpha \propto v \cdot l_{P h}
$$

without an explicit dependence on the heat capacity as in expression 11. Since the average phonon group velocity is almost independent of the temperature, it is straightforward to assume that the temperature dependence of the thermal diffusivity is related to a slowly decreasing mean free path with increasing temperature, i.e., it follows:

$$
l_{P h} \propto T^{-p}
$$

as already discussed for the temperature dependence of the thermal diffusivity.

For the calculation of the mean free path using Eq. 11 the sound velocity $\mathrm{v}$ was calculated from density and elastic modulus data $[45,46]$ which was measured by an indentation technique. The calculated values of sound velocity and phonon mean free path $l_{P h}$ at room temperature are also shown in Table 1 together with data of the density and the elastic modulus. Under the assumption that the sound velocity is nearly independent of the temperature and the phonon mean free path (Eq. 13) follows the temperature dependence of the thermal diffusivity (Eq. 12), the thermal conductivity can be modeled according the Debye kinetic theory [44] given in Eq. 11. The results of this calculation are shown by the straight lines in Fig. 4.

It appears to be very interesting that the estimated room temperature values of the phonon mean free path derived from the thermal conductivity data can be related at least approximately to characteristic atomic distances within the NASICON framework. The Na-Na atomic distances in NATP are varying between $3.2 \AA$ and $4.6 \AA$ [35], respectively, and it is important to note that completely filled $\mathrm{Na}$ sites are surrounded by only partially filled sites. Therefore, an estimated phonon mean free path of $4.6 \AA$ reflects the fact that the phonon propagation and consequently the thermal transport is also affected by this particular defect arrangement. The same effects can be observed in NaZSiP systems, in which the shortest hopping distance [23] between two Na sites varies between $3.5 \AA$ and $3.7 \AA$. However, the Na-O distance [15] are showing variations from $2.4 \AA$ to $3.1 \AA$. The estimations for the phonon mean free path is about $3.0 \AA$ in $\mathrm{Na}_{3.0} \mathrm{ZSiP}$ and $2.6 \AA$ in $\mathrm{Na}_{2.7} \mathrm{ZSiP}$. Also, within this system the phonon mean free path seems to reflect structural details of the NASICON framework.

Although a direct comparison with other literature data of the NATP or the $\mathrm{NaZSiP}$ systems is not possible due the fact that thermal diffusivity or thermal conductivity data are not available up to now, the data of this work can be related 
to thermal transport studies in Na- or Li-ion-conducting phosphate-based systems. Suleiman and Lunden [47] studied the thermal conductivity and diffusivity in the ion conducting $\mathrm{Na}_{3} \mathrm{PO}_{4}$. They found low thermal conductivity values comparable with the results of this work. The thermal conductivity data between room temperature and $530{ }^{\circ} \mathrm{C}$ vary only slowly but give evidence of phase transitions in $\mathrm{Na}_{3} \mathrm{PO}_{4}$. However, since there are no data for the ionic conductivity are given, ionic and thermal transport could not be related. Petkov and Asabina [48] studied the thermophysical properties of NZP ceramics with a $\mathrm{NaZr}_{2}\left(\mathrm{PO}_{4}\right)_{3}$ type structure. Within the temperature range from RT to $400{ }^{\circ} \mathrm{C}$ they found monotonical increasing values of the thermal conductivity for $\mathrm{NaZr}_{2}\left(\mathrm{PO}_{4}\right)_{3}$ and $\mathrm{Na}_{5} \mathrm{Zr}\left(\mathrm{PO}_{4}\right)_{3}$ starting from $0.6 \mathrm{~W} \cdot \mathrm{mK}^{-1}$ and $0.4 \mathrm{~W} \cdot \mathrm{mK}^{-1}$, respectively. This low level of the thermal conductivity values may be due to the relatively high amount (25\% to $30 \%$ ) of porosity in the samples. However, the ionic conductivity was not measured. The thermal conductivity and diffusivity, respectively, was studied [49] in the $\mathrm{Li}^{+}$conducting $\mathrm{Li}_{1.3} \mathrm{Al}_{0.3} \mathrm{Ti}_{1.7}\left(\mathrm{PO}_{4}\right)_{3}$ analog system to NATP. The ionic conducting ceramic electrolyte was consolidated by a field-assisted sintering technique. The thermal transport parameter showed a strong dependence on the sintering temperature. Within the range of sintering temperatures from $800{ }^{\circ} \mathrm{C}$ to $1000{ }^{\circ} \mathrm{C}$ the values increased by a factor of more than two which could be related to growing grain sizes and increasing density. An investigation of the thermophysical properties [41] of $\mathrm{Li}^{+}$conducting $\mathrm{Li}_{1+x} \mathrm{Al}_{x} \mathrm{Ge}_{2-x}\left(\mathrm{PO}_{4}\right)_{3}$ with $x=0.3,0.5$ and 0.7 , respectively, came to similar results as in this work for the $\mathrm{Na}^{+}$conducting $\mathrm{NaZSiP}$ system. While the ionic conductivity increased with the $\mathrm{Li}^{+}$doping level, the thermal conductivity decreases accordingly.

\section{Conclusion}

In this work, we have studied the ionic and thermal transport properties of two different $\mathrm{Na}^{+}$ion conducting NASICON-structured systems. The thermal diffusivity and the thermal conductivity, respectively, were measured for the first time within these ion conductors together with the ionic conductivity. The ionic conductivity in NaZSiP is more than two orders of magnitude higher compared to NATP. However, the values of the thermal diffusivity and the thermal conductivity of NATP are about two times higher than in NaZSiP. Obviously, the defect concentration and the vacancy arrangement seem to play an important role for both the ionic and thermal transport, respectively. While a balanced vacancy configuration, i.e., a framework of partially occupied lattice sites is crucial for the migration of the ions, it has the opposite effect on the thermal transport since increasing defect concentrations are leading to a stronger scattering of phonons and reducing the thermal conductivity.

Acknowledgements This research was funded by the German Research foundation (DFG) under the Project ID 39084152 (POLiS Cluster of Excellence). This work contributes to the research performed at CELEST (Center of Electrochemical Storage Ulm-Karlsruhe) 
Funding Open Access funding enabled and organized by Projekt DEAL.

Open Access This article is licensed under a Creative Commons Attribution 4.0 International License, which permits use, sharing, adaptation, distribution and reproduction in any medium or format, as long as you give appropriate credit to the original author(s) and the source, provide a link to the Creative Commons licence, and indicate if changes were made. The images or other third party material in this article are included in the article's Creative Commons licence, unless indicated otherwise in a credit line to the material. If material is not included in the article's Creative Commons licence and your intended use is not permitted by statutory regulation or exceeds the permitted use, you will need to obtain permission directly from the copyright holder. To view a copy of this licence, visit http://creativecommons.org/licen ses/by/4.0/.

\section{References}

1. W. Zhao, J. Yi, P. He, H. Zhou, Electrochem. Energy Rev. 2, 574 (2019)

2. Z. Zhang, Y. Shao, B. Lotsch, Y. Hu, H. Li, J. Janek, L. Nazar, C. Nan, J. Maier, M. Armand, L. Chen, Energy Environ. Sci. 11, 1945 (2018)

3. R. Chen, W. Qu, X. Guo, L. Li, F. Wu, Mater. Horiz. 3, 487 (2016)

4. M. Armand, P. Axmann, D. Bresser, M. Copley, K. Edstöm, C. Ekberg, D. Guyomard, B. Lestriez, P. Novak, M. Petranikova, W. Porcher, S. Trabesinger, M. Wolfahrt-Mehrens, H. Zhang, J. Power Source 479, 228708 (2020)

5. T. Ely, D. Kamzabek, D. Chakraborty, Front. Energy Res. 7, 71 (2019)

6. D. Karabelli, S. Singh, S. Kiemel, J. Koller, A. Konarov, F. Stubhan, R. Miehe, M. Weber, Z. Balkenov, K. Birke, Front. Energy Res. 8, 605129 (2020)

7. P. Adelhelm, P. Hartmann, C. Bender, M. Busche, C. Eufinger, J. Janek, J. Beilstein, Nanotechnology 6, 1016 (2015)

8. Q. Ma, F. Tietz, Chem. Electro. Chem. 7, 2693 (2020)

9. T. Tiefeng, B. Wang, X. Gu, L. Wang, M. Ling, G. Liu, Nano Energy 30, 756 (2016)

10. G. Sundar, G. Suman, K. Kumar, D. Dutta, R. Rao, J. Phys. Chem. Solids 126, 209 (2019)

11. M. Guin, F. Tietz, J. Power Source 272, 1036 (2015)

12. A. Nieto-Munoz, J. Ortiz-Mosquera, A. Rodrigues, J. Alloys Comp. 820, 153148 (2020)

13. J. Goodenough, H. Hong, J. Kafalas, Mat. Res. Bull. 11, 203 (1976)

14. M. Barj, H. Perthuis, P. Colomban, Solid State Ionics 9-10, 845 (1983)

15. J. Boilot, G. Colin, P. Colomban, J. Sol. State Chem. 73, 160 (1988)

16. H. Bradtmueller, A. Nieto-Munez, J. Ortiz-Mosquera, A. Rodrigues, H. Eckert, J. Non-Cryst, Solvents 489, 91 (2019)

17. S. Lunghammer, Q. Ma, D. Rettenwander, I. Hanzu, F. Tietz, H. Wilkening, Chem. Phys. Lett. 701, 147 (2019)

18. T. Tsujimura, J. Mater. Sci. 50, 7735 (2015)

19. J. Maier, U. Warhus, E. Gmelin, Sol. State Chem. 18-19, 969 (1986)

20. U. Warhus, J. Maier, A. Rabenau, Sol. State Chem. 72, 113-125 (1988)

21. L. Abello, K. Chhor, M. Barj, C. Pommier, C. Delmas, J. Mater. Sci. 24, 3380 (1989)

22. V. Petkov, E. Asabina, A. Martin, K. Kiryanov, Thermochim. Acta 403, 185 (2003)

23. H. Hong, Res. Bull. 11, 173-182 (1976)

24. A. Ignaszak, P. Pasierb, R. Gajerski, S. Komornicki, Thermochim. Acta 426, 7 (2005)

25. S. Naquash, Q. Ma, F. Tietz, O. Guillon, Sol. State Ionics 302, 83 (2017)

26. J.R. MacDonald (ed.), Impedance Spectroscopy (Wiley, Mew York, 1987)

27. K. Funke, Sol. State Ionics 94, 27 (1997)

28. JCGM 100: 2008, Evaluation of Measurement Data: Guide to the Expression of Uncertainty in Measurement (Bureau International des Poids et Mesures, Sevre)

29. P.G. Klemens, Phys. Rev. 119, 507 (1960)

30. W. Parker, R. Jenkins, C. Butler, G. Abbott, J. Appl. Phys. 32, 1679 (1961)

31. L. Vozar, Flash Method for the Thermal Diffusivity Measurement: Theory and Praxis (UKF, Nitra, 2001)

32. L. Dusza, High Temp. High Press. 27/28, 467 (1995/1996) 
33. G. Della Gatta, M. Richardson, S. Sarge, S. Stolen, Pure. Appl. Chem. 78, 1455 (2006)

34. G. Höhne, W. Hemminger, H. Flamersheim, Differential Scanning Calorimetry (Springer, Berlin, 2010)

35. D. Ditmars, S. Ishihara, S. Chang, G. Bernstein, E. West, J. Res. Nat. Bur. Stand. 87, 159 (1982)

36. F. Mouahid, M. Bettach, M. Zahir, P. Moldonado-Manso, S. Bruque, E. Losilla, M. Aranda, J. Mat. Chem. 10, 2746 (2000)

37. M. Rohde, B. Schulz, in Thermal Conductivity. ed. by J. Cremers, H. Fine (Plenum Press, New York, 1990), pp. 509-520

38. F. Sudreau, D. Petit, J.P. Boilot, J. Sol. State Chem. 83, 78 (1989)

39. J.P. Boilot, G. Collin, R. Comes, Sol. State Ionics 9-10, 829 (1983)

40. F. Tietz, A.I.M.S. Mat, Science 4, 1305 (2017)

41. M. Rohde, Y. Cui, C. Ziebert, H. Seifert, Int. J. Thermophys. 41, 31 (2020)

42. J. Rodrigo, J. Alamo, Mat. Res. Bull. 26, 475 (1991)

43. N. Dhas, K. Patil, J. Mater. Chem. 4, 491 (1994)

44. R. Peierls, Ann. Phys. 3, 1055 (1929)

45. J. Nonemacher, S. Naquash, F. Tietz, J. Malzbender, Ceramic Int. 45, 21308 (2019)

46. W. Oliver, G. Pharr, J. Mater. Res. 7, 1564 (1992)

47. B. Suleiman, A. Lunden, J. Phys. Condens. Mater. 15, 6911 (2003)

48. V. Petkov, E. Asabina, Glass Ceram. 61, 233 (2004)

49. E. Bucharsky, K. Shell, T. Hupfer, M. Hoffmann, M. Rohde, H.J. Seifert, Ionics 22, 1043 (2016)

Publisher's Note Springer Nature remains neutral with regard to jurisdictional claims in published maps and institutional affiliations. 